Vibration and symmetry-breaking of boron nitride nanotubes

This article has been downloaded from IOPscience. Please scroll down to see the full text article.

2010 Nanotechnology 21365702

(http://iopscience.iop.org/0957-4484/21/36/365702)

View the table of contents for this issue, or go to the journal homepage for more

Download details:

IP Address: 137.44.1.174

The article was downloaded on 22/09/2010 at 13:17

Please note that terms and conditions apply. 


\title{
Vibration and symmetry-breaking of boron nitride nanotubes
}

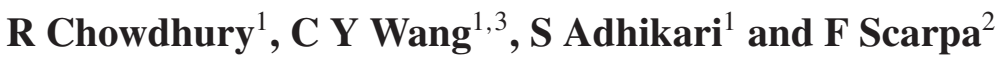 \\ ${ }^{1}$ School of Engineering, Swansea University, Singleton Park, Swansea SA2 8PP, UK \\ ${ }^{2}$ Advanced Composites Centre for Innovation and Science, University of Bristol, \\ BS8 1TR, UK \\ E-mail: chengyuan.wang@swansea.ac.uk
}

Received 18 May 2010, in final form 3 July 2010

Published 11 August 2010

Online at stacks.iop.org/Nano/21/365702

\begin{abstract}
The unique features of axial, torsional, transverse and radial breathing vibrations are captured for armchair and zigzag singlewalled boron nitride nanotubes (BNNTs) based on molecular mechanics simulations and continuum mechanics theories. Equivalent Young's modulus $1 \mathrm{TPa}$ and shear modulus $0.4 \mathrm{TPa}$ are obtained independent of the chirality of BNNTs. In particular, a distorted optimized structure is observed for the first time for BNNTs with sufficiently large diameter and length. It is found that the deformed structures result in behaviours of BNNTs deviating from those of classical columns/beams. Such symmetry-breaking could also exert significant impacts on the structural instability (buckling) and electronic properties of BNNTs that are sensitive to the structural symmetry.
\end{abstract}

(Some figures in this article are in colour only in the electronic version)

\section{Introduction}

After the discovery of carbon nanotubes (CNTs) in experimental studies [1,2], Blase et al [3] theoretically predicted the possibility of obtaining boron nitride nanotubes (BNNTs) and initiated an investigation on their distinctive properties $[4,5]$ by exploring the similarity between hexagonal boron nitride (h-BN) sheets [6] and graphite. An h-BN sheet is composed of alternating atoms of boron and nitrogen but $\mathrm{BN}$ is mostly found in the same phases, which produces atomic structures similar to those of graphite. It is noted that even the crystallographic parameters of h-BN sheets and graphite are almost equal. Rolling up an h-BN sheet in different chiral directions generates BNNTs of various chiralities, such as zigzag, chiral and armchair. Such BNNTs possess many superior properties similar to those of CNTs [7,8], such as exceptional elastic properties [9-15], high mechanical strength [16-21], chemical inertness [22], high heat conduction and piezoelectricity [23]. On the other hand, different from CNTs which are highly toxic to the human body and, depending on their chirality, can be semiconductors and conductors BNNTs of different chirality show good biocompatibility and are always semiconducting with a large band gap [4].

\footnotetext{
3 Author to whom any correspondence should be addressed.
}

Up to now, a number of investigations have been reported on the mechanical properties of BNNTs [9-21]. In experiments, Chopra and Zettl [19] determined that the Young's modulus of multi-walled BNNTs is $1.22 \pm 0.24 \mathrm{TPa}$, while Suryavanshi et al [18] measured the average value $0.722 \mathrm{TPa}$, with an error of $\pm 20 \%$. In theoretical predictions, Hernandez et al's tight-binding calculation [24] predicted a Young's modulus of singlewalled BNNTs of 0.837-0.912 TPa almost independent of their diameter. Similar observation was made by Akdim et al [25] with a lower value of Young's modulus. The vibrational properties of singlewalled BNNTs were also examined in previous studies. Based on a tight-binding method, Sanchez-Portal and Hernandez [26] determined the diameter-dependency of the frequency for the radial breathing mode of singlewalled BNNTs. Wirtz and Rubio [27] calculated the phonon-dispersion relation for Raman and infrared active modes of zigzag and armchair BNNTs using first principles. A comparative study of the radial breathing mode was also conducted in [25] showing that the frequencies of BNNTs are lower than those of CNTs whose diameter is close to that of BNNTs. In addition, the surface buckling that is unique to BNNTs was observed as a result of the different hybridizations of boron and nitrogen in the curved nanotube surfaces [10, 24, 27, 28]. 
Nevertheless, a systematic study on various BNNT vibrations has not yet been available, in spite of the fact that such an investigation is essential for a wide range of the applications of BNNTs, e.g., nanoresonators, nanosensors, actuators and transducers [29-33]. In particular, singlewalled BNNTs so far have been considered as circular nano-cylinders like singlewalled CNTs. This, however, may not be always true as the iconicity of $\mathrm{B}-\mathrm{N}$ bonds responsible for the surface buckling in hoop direction could also lead to distortion of the optimized configuration in the axial direction when BNNTs are used with practical end constraints. This issue has to be clarified as the geometry of optimized BNNTs can have strong impacts on their mechanical behaviours and the physical properties that are sensitive to the geometric symmetry.

Motivated by these ideas, the present work aims to achieve a realistic configuration of optimized singlewalled BNNTs and conduct a detailed study on various vibrations of such BNNTs. This study also offers a useful probe for fundamental elastic properties and overall mechanical behaviours of BNNTs. Here BNNTs will be formed and optimized using molecular mechanics (MM) simulations based on a universal force field (UFF). The data obtained in MM simulations will be further examined and interpreted based on continuum mechanics theories.

\section{Molecular mechanics modelling}

Molecular mechanics is an empirical technique to optimize structures with high accuracy and comparably little computational expense. Simulations were performed with Gaussian [34], using the general purpose universal force field (UFF) [35]. The UFF is parameterized for the full periodic table and investigated for structures of main group, organic, transition metal inorganic, and organometallic compounds. All the force field parameters include a set of hybridization, effective nuclear charges, and connectivity. The UFF is well suited for dynamics simulations because it allows more accurate vibration measurements than many other force fields, which do not distinguish bond strengths. The UFF and parameters for boron and nitrogen have been described in detail in [35]. The UFF is a purely harmonic force field with a potential-energy expression of the form:

$E=\sum E_{R}+\sum E_{\theta}+\sum E_{\phi}+\sum E_{\omega}+\sum E_{\mathrm{VDW}}+\sum E_{\mathrm{el}}$.

The valence interactions consist of bond stretching $\left(E_{R}\right)$, which is a harmonic term, and angular distortions. The angular distortions are bond angle bending $\left(E_{\theta}\right)$, described by a three term Fourier cosine expansion, dihedral angle torsion $\left(E_{\phi}\right)$ and inversion terms (out-of-plane bending) $\left(E_{\omega}\right) . E_{\phi}$ and $E_{\omega}$ are described by cosine-Fourier expansion terms. The non-bonded interactions consist of van der Waals $\left(E_{\mathrm{VDW}}\right)$ and electrostatic $\left(E_{\mathrm{el}}\right)$ terms. $E_{\mathrm{VDW}}$ is described by a Lennard-Jones potential and $E_{\mathrm{el}}$ is described by a Columbic term. The functional form of the above energy terms is given as follows:

$$
\begin{gathered}
E_{R}=k_{1}\left(r-r_{0}\right)^{2} \\
E_{\theta}=k_{2}\left(C_{0}+C_{1} \cos \theta+C_{2} \cos 2 \theta\right) \\
C_{2}=\frac{1}{4 \sin ^{2} \theta} \quad C_{1}=-4 C_{2} \cos \theta_{0} \\
C_{0}=C_{2}\left(2 \cos ^{2} \theta_{0}+1\right) \quad E_{\phi}=k_{3}(1 \pm \cos n \phi) \\
E_{\omega}=k_{4}\left(1 \pm \cos \left(n \chi-\chi_{0}\right)\right) \\
E_{\mathrm{VDW}}=D\left[\left(\frac{r^{*}}{r}\right)^{12}-2\left(\frac{r^{*}}{r}\right)^{6}\right] \quad E_{\mathrm{el}}=\frac{q_{\mathrm{i}} q_{j}}{\varepsilon r_{i j}} .
\end{gathered}
$$

Here $k_{1}, k_{2}, k_{3}$ and $k_{4}$ are force constants, $\theta_{0}$ is the natural bond angle, $D$ is the van der Waals well depth, $r^{*}$ is the van der Waals length, $q_{\mathrm{i}}$ is the net charge of an atom, $\varepsilon$ is the dielectric constant and $r_{i j}$ is the distance between two atoms. In addition, $E_{\mathrm{el}}$ is a Columbic term which is nonzero in BNNTs. The atomic charges are assigned according to the 'Qeq algorithm' presented in [36]. The values of these parameters in equations (2) can be found in [35].

Here we should point out that for small diameters, the hybridization of the electronic structure has been observed in BNNTs using DFT simulations, with band gap values lower in smaller $(3,0)$ and $(4,0)$ models compared with ultralarge ones due to $\pi-\Omega$ hybridization [37]. Also while for most DFT calculations the average bond length is reported at $1.44 \AA$ [38], values between 1.47 and $1.48 \AA$ have been observed for small diameters [37]. The different average bond lengths would provide a mechanical anisotropy along axial and circumferential directions, which the MM model (not based on quantum mechanics principles) would tend to underestimate. However, the equivalent mechanical anisotropy of the BNNTs would affect principally the modes of higher order, rather than the global bending-dominated ones.

\section{Results and discussion}

Based on the aforementioned MM model, we simulate the vibrational behaviours of armchair and zigzag BNNTs. The phenomena observed in MM simulations will be further analysed by using continuum mechanics theories. In addition, the values of equivalent elastic properties will be extracted via the comparison between the MM model and the continuum models.

\subsection{Axial and torsional vibration}

In this section we first explore the axial and torsional vibrations of the BNNTs that are clamped at their two ends. Two types of tubes are considered, namely armchair tubes $(3,3),(4,4)$ and $(6,6)$ whose diameter increases from 0.413 to $0.551 \mathrm{~nm}$ and $0.826 \mathrm{~nm}$, and zigzag tubes $(5,0),(8,0)$ and $(10,0)$ where the diameter grows from 0.389 , to $0.636 \mathrm{~nm}$ and $0.795 \mathrm{~nm}$.

Our MM simulations show that in the axial vibration (figure 1(a)) all the boron and nitrogen atoms of BNNTs oscillate along the longitudinal direction. The tube crosssection retains its original shape and vibrates as a rigid body 


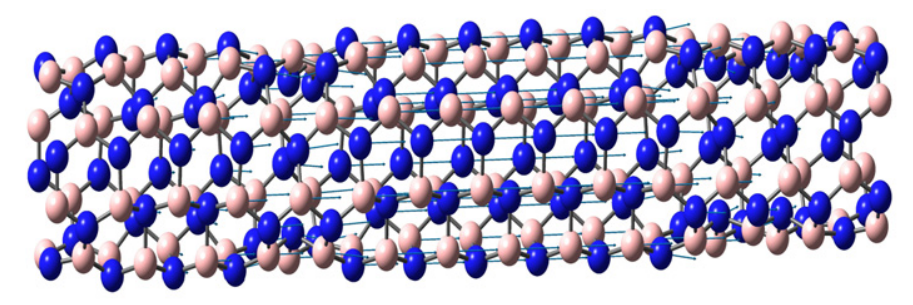

(a)

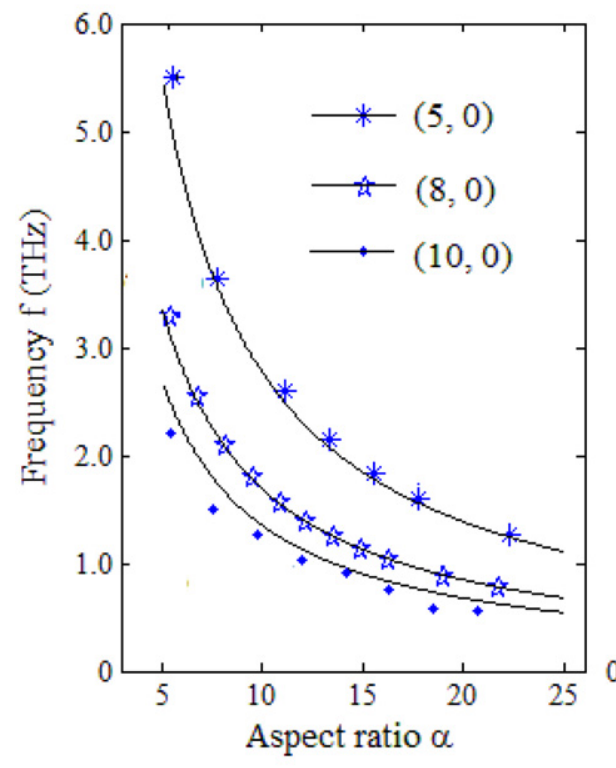

(b)

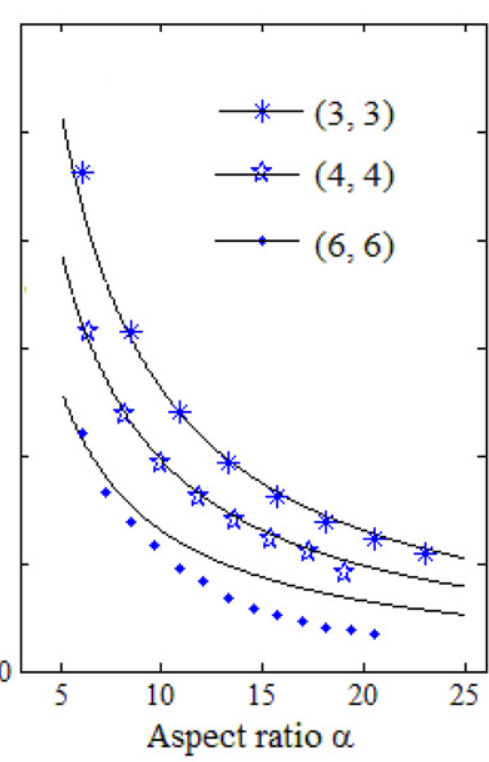

(c)

Figure 1. (a) Axial vibration and its associated frequency of (b) zigzag and (c) armchair BNNTs given by the MM simulations (discrete dots) and a column model with Young's modulus 1 TPa (solid lines).

in the axial direction. The maximum axial displacement is obtained for the cross-section in the middle of the tubes. It declines gradually when the cross-section moves away from the centre and becomes zero at the two clamped ends. The axial strain is thus generated due to such a non-uniformly distributed displacement. The frequency of the axial vibration is shown in figures 1(b) and (c) against the (length-to-diameter) aspect ratio $\alpha$ for zigzag and armchair BNNTs, respectively. From the figure it is seen that the frequency falls in the range of 0.6$5.5 \mathrm{THz}$ for the BNNTs whose aspect ratio $\alpha$ varies between 6 and 24. For each BNNT (i.e., fixed diameter) the axial mode frequency decreases substantially with the increasing aspect ratio $\alpha$ or increasing length. In the meantime, for a given $\alpha$ the frequency of axial vibration also declines when the tube diameter grows. For example, figure 1(b) shows that, for $\operatorname{BNNT}(3,3)$ the frequency of axial mode decreases from 4.6 to $1 \mathrm{THz}$ when the aspect ratio increases from 6 to 23 . Also, at $\alpha \approx 8.5$ the frequency of $\operatorname{BNNT}(3,3)$ is $3.2 \mathrm{THz}$, two times as much as $1.6 \mathrm{THz}$ of $\operatorname{BNNT}(6,6)$.

Torsional vibration (figure 2(a)) has also been achieved in the MM simulations for the clamped BNNTs. In this mode, the boron and nitrogen atoms of the BNNTs oscillate in the tangential direction of the cross-section perimeter. However the relative position of the atoms on the same cross-section does not change during the vibration. In other words, the cross-section rotates back and forth about the axis of the tubes as a rigid body. The (angular) displacement of the crosssection varies along the axial direction in such a way that it increases monotonically from the minimum value (zero) at the two clamped ends to the maximum value at the centre of the BNNTs. The torsional strain therefore occurs for BNNTs along the axial direction. The frequency of the torsional mode is calculated in figures 2(b) and (c), respectively, for zigzag and armchair BNNTs with the aspect ratio increasing from around 6 to 23. It is shown in the figure that, similar to the axial vibration, torsional vibration frequency decreases with the increasing aspect ratio for both armchair and zigzag BNNTs. In addition, for a given aspect ratio, the frequency also declines with the increasing diameter. The highest frequency $3.4 \mathrm{THz}$ is obtained for BNNT $(5,0)$ of aspect ratio 5.6 and the lowest frequency $0.13 \mathrm{THz}$ is achieved for BNNT $(6,6)$ of aspect ratio 21 .

The above results show that, in the axial and torsional vibrations, the behaviours of discrete BNNTs are quite close to those of continuum bars/columns. Thus we are particularly interested in comparing the MM simulations with 


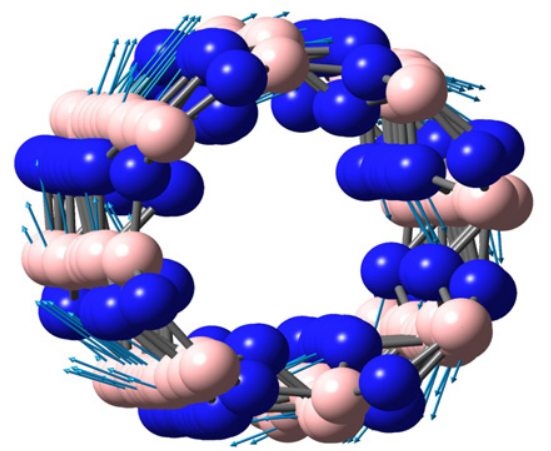

(a)

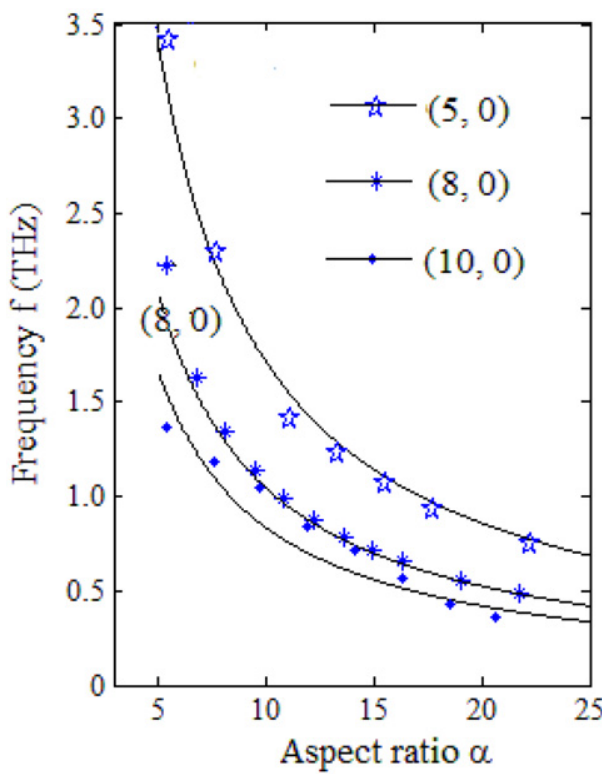

(b)

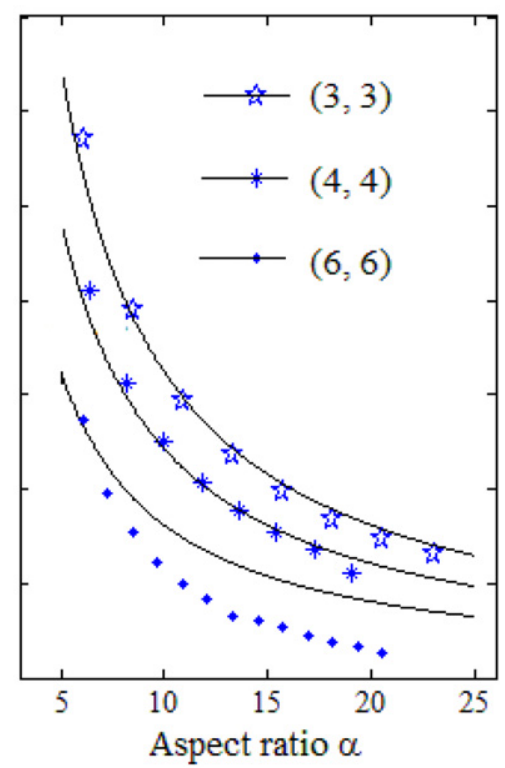

(c)

Figure 2. (a) Torsional vibration and its associated frequency of (b) zigzag and (c) armchair BNNTs given by the MM simulations (discrete dots) and a column model with shear modulus $0.41 \mathrm{TPa}$ (solid lines).

the continuum bar/column models. The study could also offer an opportunity to extract the values of the elastic constants of BNNTs and bring in better understanding of the MM simulations. For a clamped column the frequencies of axial $\left(f_{\mathrm{A}}\right)$ and torsional $\left(f_{\mathrm{T}}\right)$ vibrations are given by [39]

$$
f_{\mathrm{A}}=\left(\frac{E}{\rho}\right)^{1 / 2}\left(\frac{n}{2 L}\right) \quad \text { and } \quad f_{\mathrm{T}}=\left(\frac{G}{\rho}\right)^{1 / 2}\left(\frac{n}{2 L}\right) \text {. }
$$

Here $n$ is the half axial wavenumber of vibration, $L$ is the length of columns, $E$ is Young's modulus, $G$ is shear modulus and $\rho$ is the mass density of the material that the column is made of. Equation (3) shows that the axial and torsional frequencies of columns are functions of the length $L$. However the diameter and the aspect ratio are not included in the equation. To compare the column model with the MM simulations in figures 1 and 2 we express the frequencies in terms of the diameter and aspect ratio of a column and set $n=1$ as observed in the simulations.

$$
f_{\mathrm{A}}=\frac{v_{\mathrm{A}}}{2}\left(\frac{1}{d}\right)\left(\frac{1}{\alpha}\right) \quad \text { and } \quad f_{\mathrm{T}}=\frac{v_{\mathrm{T}}}{2}\left(\frac{1}{d}\right)\left(\frac{1}{\alpha}\right)
$$

where $v_{\mathrm{A}}=\left(\frac{E}{\rho}\right)^{1 / 2}$ and $v_{\mathrm{T}}=\left(\frac{G}{\rho}\right)^{1 / 2}$ give the propagation speeds of the axial and torsional wave in the axial direction of the column. It is easy to see that equation (4) is equivalent to equation (3). Subsequently, we have fitted equation (4) with different values of $v_{\mathrm{A}}$ and $v_{\mathrm{T}}$ to the data displayed in figures 1 and 2 for axial and torsional vibrations of the BNNTs. The curves associated with $v_{\mathrm{A}}=2.15 \times 10^{4} \mathrm{~m} \mathrm{~s}^{-1}$ and $v_{\mathrm{T}}=$ $1.33 \times 10^{4} \mathrm{~m} \mathrm{~s}^{-1}$ show the best fit of the column model to the MM simulations for BNNTs with relatively small diameter (i.e., $(3,3),(4,4),(5,0)$ and $(8,0))$. However, for BNNTs with relatively large diameter (i.e., $(6,6)$ and $(10,0))$ the two models do not match. To examine this issue, in what follows we shall classify the BNNTs studied here into two categories, namely (1) small-diameter BNNTs, i.e., armchair tubes $(3,3)$ 
and $(4,4)$ and zigzag tubes $(5,0),(8,0)$, and (2) (relatively) large-diameter BNNTs, i.e., armchair tube $(6,6)$ and zigzag tube $(10,0)$.

3.1.1. Small-diameter BNNTs. First let us look at the BNNTs with a small diameter. As shown in figures 1 and 2 , the column model with the chosen values of material constants is consistently in good agreement with the MM simulations for the axial and torsional vibrations of armchair (i.e., (3, $3),(4,4))$ and zigzag (i.e., $(5,0),(8,0))$ BNNTs whose diameter changes from 0.389 to $0.636 \mathrm{~nm}$ and aspect ratios from around 6 to 23. These results show clear evidence that when the axial and torsional vibrations are concerned, the small-diameter BNNTs comprising boron and nitrogen atoms are approximately equivalent to continuum columns made of a homogeneous and isotropic material. The chirality of the BNNTs does not exert significant impacts on the overall mechanical behaviours of these BNNTs.

It is noted that the arithmetic average of boron and nitride atomic masses is equal to carbon atomic mass, and similar to the carbon atoms in SWCNTs, all the boron and nitride atoms of singlewalled BNNTs are in hexagonal arrangement where the $\mathrm{B}-\mathrm{N}$ bond length is around $0.144 \mathrm{~nm}$, very close to $0.142 \mathrm{~nm}$ of $\mathrm{C}-\mathrm{C}$ bond length in SWCNTs. Thus, the diameter of singlewalled BNNTs is approximately equal to that of SWCNTs of the same chirality. In addition, in the present study we consider the BNNTs whose diameter is not smaller than that of BNNTs $(4,0)$. The displacement of surface buckling for such BNNTs is not larger than $0.01 \mathrm{~nm}[24,28]$ which is less than $3 \%$ of the average radius. Thus, the surface buckling cannot significantly affect the average diameter of these BNNTs. It follows that when the same effective thickness $h=0.34 \mathrm{~nm}$ is used the mass density $\rho_{\mathrm{bn}}$ of singlewalled BNNTs should be very close to $2.27 \mathrm{~g} \mathrm{~cm}^{-3}$ of SWCNTs [40]. Using $\rho_{\mathrm{bn}}=2.27 \mathrm{~g} \mathrm{~cm}^{-3}$, the above values of $v_{\mathrm{A}}$ and $v_{\mathrm{T}}$ lead to equivalent Young's modulus $E \approx 1 \mathrm{TPa}$ and equivalent shear modulus $G \approx 0.4 \mathrm{TPa}$ for the singlewalled BNNTs. Here it should be pointed out that $E \approx 1 \mathrm{TPa}$ and $G \approx$ $0.42 \mathrm{TPa}$ obtained for singlewalled BNNTs are associated with $h=0.34 \mathrm{~nm}$. These values given by the present MM simulations are found to be in accordance with those measured in experiments [19], i.e., $E=1.2 \pm 0.24 \mathrm{TPa}$, and by a first principle method [24], i.e., $E=0.7-0.9 \mathrm{TPa}$ and $G=$ $0.3 \mathrm{TPa}$, with the same effective thickness $h$.

Furthermore, as shown in equations (3), for BNNTs as equivalent columns the frequencies of both axial and torsional vibrations are determined by their length and the material properties. The diameter of BNNTs/column however has no effects on the frequencies of the two modes. The diameterdependence of the frequency shown in figures 1 to 2 and equations (4) is due to the fact that for the BNNTs (or columns) of the same aspect ratio the increase/decrease of their diameter is always accompanied by the growth/reduction of their length. Nevertheless, if the length of BNNTs is fixed the identical frequency of the axial or torsional vibration can be obtained for BNNTs of different diameter. This behaviour of the axial and torsional vibrations can be explained by the unique feature observed in the MM simulations that, in these two modes

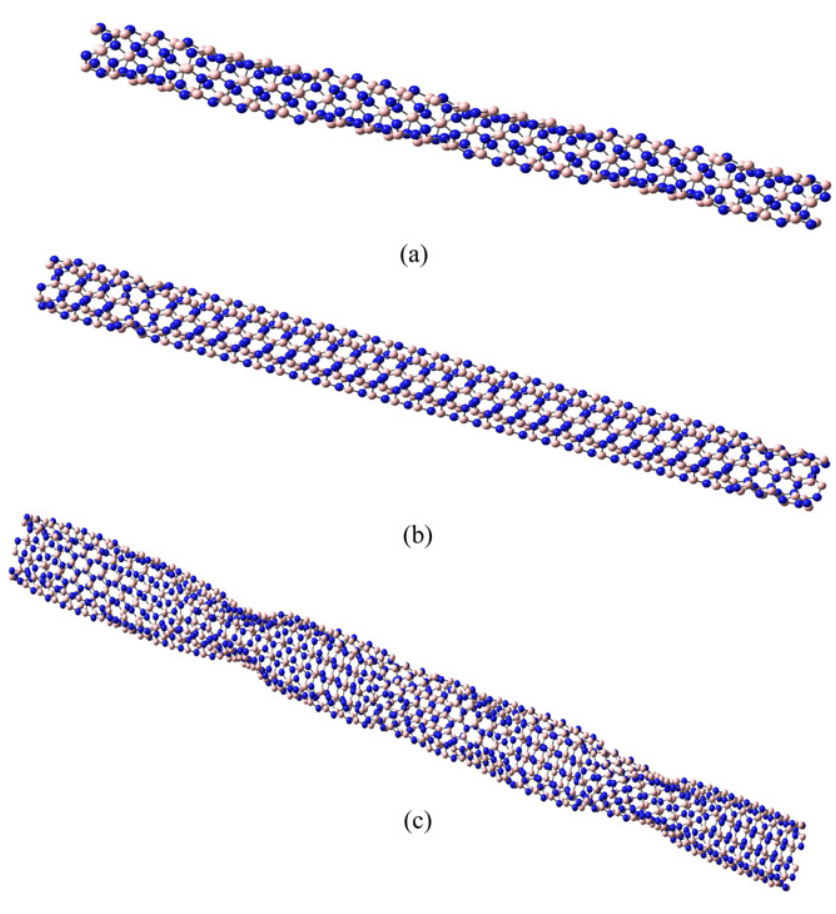

Figure 3. Optimized configuration of armchair BNNTs: (a) $(3,3)$, (b) $(4,4)$ and (c) $(6,6)$ with the aspect ratio 15 .

the cross-sections of BNNTs only have a rigid body motion without any radial/circumferential distortion. The strain of BNNTs during the vibrations is solely determined by the rate of changes in the axial or angular displacements of the rigid cross-section along the axial direction.

3.1.2. (Relatively) large-diameter BNNTs. Next we shall study the BNNTs with a relatively large diameter, i.e., armchair tube $(6,6)$ and zigzag tube $(10,0)$. In figures 1 and 2 , large discrepancy is found between the MM simulations and the column model for the two BNNTs, in spite of the fact that such a column model matches the MM simulations very well for the small-diameter BNNTs. To understand the observation we have examined the geometric configuration of the optimized BNNTs in their equilibrium state. The images of BNNTs (3, $3),(4,4)$ and $(6,6)$ and BNNTs $(5,0),(8,0)$ and $(10,0)$ are presented in figures 3 and 4 , respectively. It is seen from the figures that BNNTs $(3,3),(4,4),(5,0)$ and $(8,0)$ with diameter $<0.65 \mathrm{~nm}$ look like columns with almost uniform circular cross-sections. For each tube, the diameter of the cross-section remains approximately constant throughout the whole length of the tubes. In contrast, optimized BNNTs $(6,6)$ and $(10,0)$ with relatively large diameter exhibit distorted cross-sections whose size varies substantially along the axial direction. From this observation it follows that the BNNTs with diameter larger than $0.65 \mathrm{~nm}$ cannot be considered as uniform columns. Specifically such distortion found in optimized BNNTs could also lead to the changes in the bond length and bond angles, and finally, result in alteration of the material properties of BNNTs, such as Young's modulus and shear modulus. This symmetry-breaking of (relatively) large-diameter BNNTs observed in figures 3 and 4 therefore should be responsible for the large discrepancy between the MM simulations and 


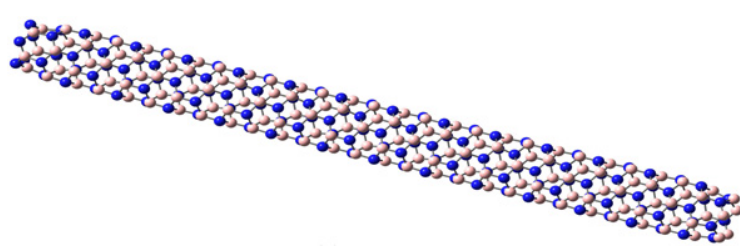

(a)

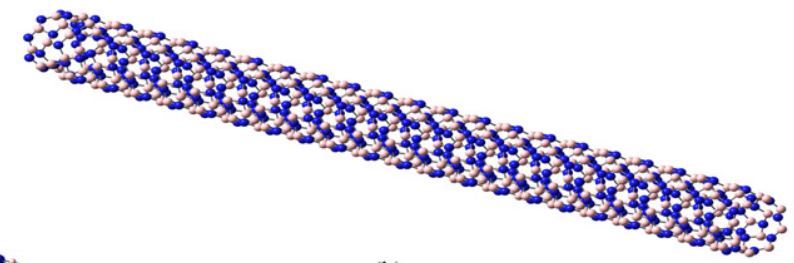

(b)

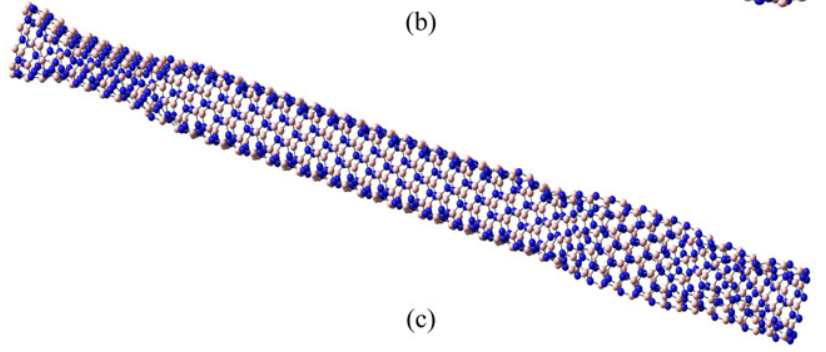

Figure 4. Optimized configuration of zigzag BNNTs: (a) $(5,0)$, (b) $(8,0)$ and $(c)(10,0)$ with the aspect ratio 15 .

the uniform column models shown in figures 1 and 2 . To the best of our knowledge, such symmetry-breaking has not been reported in the literature so far for BNNTs. For example, in [10] a similar MM model based on UUF was used to simulate the optimized configuration of singlewalled BNNTs, where axisymmetric structures are achieved for singlewalled BNNTs of various diameters without any distortion. The possible reason for the discrepancy between [10] and the present work is that, in [10], infinite BNNTs were considered with periodic boundary condition along the axial direction whereas in the present study finite BNNTs are studied with non-periodic boundary condition enforced on their two ends. To clarify this issue we have considered the periodic boundary condition for (relatively) large-diameter BNNTs $(10,0)$ and $(6,6)$. Perfect hollow cylindrical structures are obtained for the two BNNTs. This result shows evidence that the effect of non-periodic boundary conditions on BNNT ends is one of the reasons that is responsible for the symmetry-breaking of the BNNTs. Here it is emphasized that the symmetrybreaking is of major interest as it not only changes the dynamic behaviours of BNNTs as shown above but could also have significant influence on the structural stability/instability and electrical/electronic properties of BNNTs that are sensitive to the symmetry of tube geometry. A further investigation shows that similar to the surface buckling $[10,24,27,28]$ unique to BNNTs, the symmetry-breaking is also absent for SWCNTs in our MM simulations. We therefore believe that the iconicity of B-N bonds that leads to the surface buckling of BNNTs could also play a role in the distortion of the optimized configuration of BNNTs. Indeed, this issue deserves to be examined in more detail in future studies.

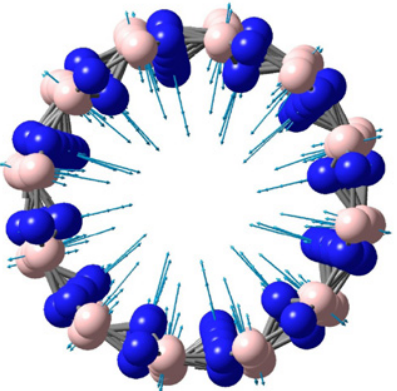

(a)

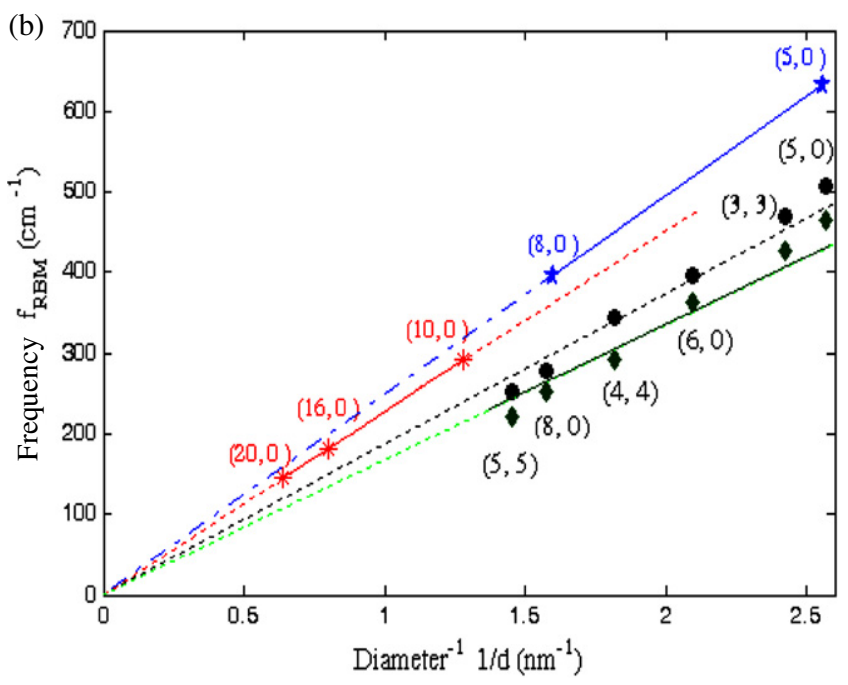

Figure 5. (a) Radial breathing mode of BNNTs and (b) the frequency of radial breathing mode against the inverse of tube diameter obtained for SWCNTs with free (asterisks) and clamped ends (stars), and BNNTs with free (diamonds) and clamped ends (circles).

\subsection{Radial breathing mode}

For SWCNTs, radial breathing vibration is characterized by the in phase radial oscillation of carbon atoms, i.e., all the atoms of an SWCNT are oscillating in radial direction synchronically as if the whole tube was breathing. The radial breathing mode $(\mathrm{RBM})$ is of major interest as it provides a useful probe for the geometric size and atomic structures of SWCNTs [41, 42]. Similar RBM has also been observed in our MM simulations for singlewalled BNNTs as shown in figure 5(a). To study RMB of BNNTs we consider armchair BNNTs (3, 3), (4, $4)$ and $(5,5)$, and zigzag tubes $(5,0),(6,0)$ and $(8,0)$, whose diameter is smaller than $0.65 \mathrm{~nm}$ and thus, as discussed above, their cross-section is approximately circular and almost uniform throughout their length. Previous studies on the RBM of SWCNTs [24, 39-43] and singlewalled BNNTs [16] have always been conducted for tubes free from tractions. However, in the application of nanotubes different end constraints can be imposed on their two ends. Specifically, in many cases the bridged nanotubes/nanowires are of practical interest. Thus, in the present study two different constraints, i.e., traction free and clamped ends, are enforced on the BNNTs. The frequencies of RBMs are shown in figure 5(b) versus the inverse of tube diameter $d$ of BNNTs. For the sake of comparison the RBM frequencies are also plotted in figure 5(b) 
for zigzag SWCNTs with free-free (FF) or clamped-clamped (CC) ends.

Figure 5(b) shows that the RBM frequencies of free SWCNTs $(10,0),(16,0)$ and $(20,0)$ represented by asterisks stay on the same straight line that passes through the origin of the coordinate. This indicates that the RBM frequency $f\left(\mathrm{~cm}^{-1}\right)$ of free SWCNTs is proportional to the inverse of their diameter $d(\mathrm{~nm})$, i.e., $f_{\mathrm{RBM}}=\frac{c_{\mathrm{cnt}}}{d}$. The coefficient $c_{\mathrm{cnt}}$ given by the present MM simulations is $227\left(\mathrm{~cm}^{-1} \mathrm{~nm}^{-1}\right)$ for free SWCNTs, which is in excellent agreement with previous studies using Raman spectroscopy [41] and various atomistic simulations [16, 43]. This result demonstrates the accuracy of the present MM simulations in studying the vibration of nanotubules. The strict linear relationship between RMB frequency and the inverse of tube diameter still holds true for the clamped SWCNTs denoted by stars in figure 5(b). The coefficient $c_{\text {cnt }}$ however rises from $227\left(\mathrm{~cm}^{-1} \mathrm{~nm}^{-1}\right)$ of free SWCNTs to $249\left(\mathrm{~cm}^{-1} \mathrm{~nm}^{-1}\right)$ of clamped tubes. It follows that the RMB of clamped SWCNTs is more sensitive to the change in diameter than that of free SWCNTs due to their enhanced radial stiffness.

For BNNTs, as shown in figure 5(b), the tendency of $f_{\mathrm{RBM}}$ to change with $d$ is generally the same as that of SWCNTs, i.e., $f_{\mathrm{RBM}} \approx \frac{c_{\mathrm{bn}}}{d}$ is observed for both FF and CC BNNTs represented by circles and diamond, respectively. Such a $f_{\mathrm{RBM}}-d$ relation is also reported in a recent study [16]. The value of coefficient $c_{\mathrm{bn}}$ is determined, which gives the best fit of the curve $f_{\mathrm{RBM}}=\frac{c_{\mathrm{bn}}}{d}$ to the data given by the MM simulations (figure 5(b)). It is found that $c_{\text {bn }}$ grows from 167 to $187\left(\mathrm{~cm}^{-1} \mathrm{~nm}^{-1}\right)$ when the free ends of BNNTs are replaced by the clamped ends. The $c_{\mathrm{bn}}$ values obtained are smaller than the corresponding $c_{\mathrm{cnt}}$ values of SWCNTs for both FF and $\mathrm{CC}$ ends. In addition, the chirality of BNNTs does not have significant influences on the diameter-dependence of $f_{\text {RBM }}$. In other words, the coefficient $c_{\text {bn }}$ is sensitive to the constraints enforced on the two ends of BNNTs but almost independent of their atomic structures. In view of these unique features it follows that the RBM of BNNTs has the potential to serve as a useful tool for the estimation of the diameter and also, the end constraints of BNNTs.

On the other hand, in figure 5(b), while $f_{\text {RBM }}$ of SWCNTs strictly abides by the linear law with $\frac{1}{d}$, relatively large deviation is seen for $f_{\mathrm{RBM}}$ of BNNTs from the linear relation. The difference can possibly be explained by the fact that the cross-section of SWCNTs consisting of carbon atoms is circular, whereas that of singlewalled BNNTs comprising both boron and nitrogen atoms is distorted to some extent due to the surface buckling [10, 27, 28, 44].

\subsection{Transverse bending modes}

Transverse bending vibrations are also observed in our MM simulations for aforementioned BNNTs with clamped ends (figure 6(a)). These include the transverse modes with one to three half waves and increasing frequencies. The lowest frequency corresponds to the mode with one half wave, and is plotted in figures $6(\mathrm{~b})$ and (c) for the zigzag and armchair BNNTs, respectively. The figure shows that, for each tube with a fixed diameter the frequency of both zigzag and armchair tubes decreases rapidly with the rising aspect ratio $\alpha$, i.e., increasing length of BNNTs. It is well known that for a clamped classical beam the frequency is calculated by [38]

$$
f=\frac{\lambda_{\mathrm{i}}}{2 \pi L^{2}}\left(\frac{E I}{\bar{m}}\right) \quad(i=1,2,3, \ldots) .
$$

Here $\lambda_{1}=4.73004074, \lambda_{2}=7.85320462$ and $\lambda_{3}=$ 10.9956079, EI is the flexural bending stiffness and $\bar{m}$ is the mass density per unit length. If we consider BNNTs as equivalent continuum beams their effective bending stiffness can be calculated based on equation (5) and the values of $f$ and $\bar{m}$ obtained in the MM simulations. Proceeding in this way we found that the equivalent bending stiffness of BNNTs increases with the growing diameter of BNNTs. For example, the mean values of $E I$ obtained from the first to the third modes rises from the order of $5.14 \times 10^{-27} \mathrm{~N} \mathrm{~m}^{2}$, to $9.79 \times 10^{-27} \mathrm{~N} \mathrm{~m}^{2}$ and to $9.37 \times 10^{-26} \mathrm{~N} \mathrm{~m}^{2}$ for BNNTs $(3,3),(4,4)$ and $(6,6)$. Similarly, it grows from the order of $4.91 \times 10^{-27}$ to $2.73 \times 10^{-26}$ to $4.84 \times 10^{-26} \mathrm{~N} \mathrm{~m}^{2}$ for BNNTs $(5,0)$, $(8,0)$ and $(10,0)$. This behaviour is qualitatively similar to continuum beams (but not exactly the same), which reveals that different from the axial and torsional vibrations independent of tube cross-sections, the transverse vibration of BNNTs is very sensitive to the change of their cross-section size. As a result, when the length of BNNTs is fixed the frequency of the same transverse modes up-shifts when the diameter of BNNTs grows.

On the other hand, it is also seen that the bending stiffness $E I$ obtained for the same BNNT varies substantially when their length changes. In this aspect, BNNTs are in contrast with the continuum beams of uniform cross-sections where the bending stiffness is independent of their length. To get some idea, the value of $E I$ obtained for the first mode of BNNT $(3,3)$ varies between $5.14 \times 10^{-27}$ and $4.28 \times 10^{-27} \mathrm{~N} \mathrm{~m}^{2}$ when the aspect ratio changes from 6 to 23. The uncertainty of $E I$ value can be possibly attributed to the distortion of BNNT crosssections induced by the surface buckling and the symmetrybreaking. It is worth mentioning that the symmetry-breaking is small for small-diameter tubes (figures 3 and 4) and thus has almost negligible effects on the axial and torsional modes that are mainly determined by tube length but insensitive to the changes of their cross-sections. Nevertheless, it could still have strong influence on the transverse modes that are much more sensitive to the variation of BNNT cross-sections. Here it should be emphasized that caution must be taken when the continuum beam, column/bar and shell models are employed to characterize the mechanical behaviours of BNNTs because BNNTs with perfectly symmetric structures may not exist in many cases. This is especially so when the mechanical properties sensitive to the variation of tube cross-section are concerned.

\section{Conclusions}

A molecular mechanics model has been used to simulate the optimized structures of singlewalled boron nitride nanotubes (BNNTs) and their vibration behaviours. Traction free and/or 


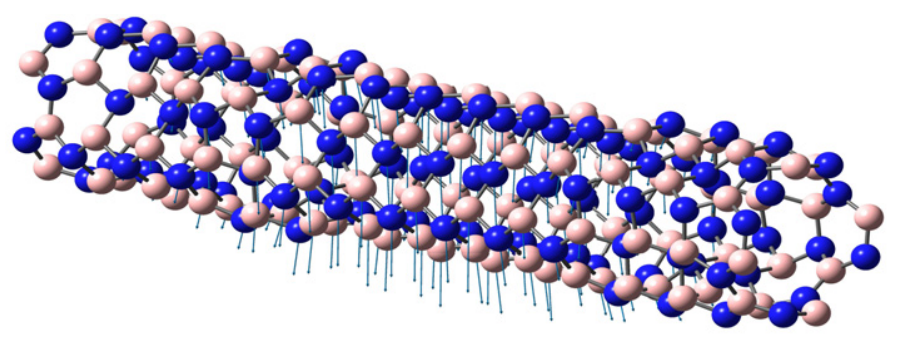

(a)

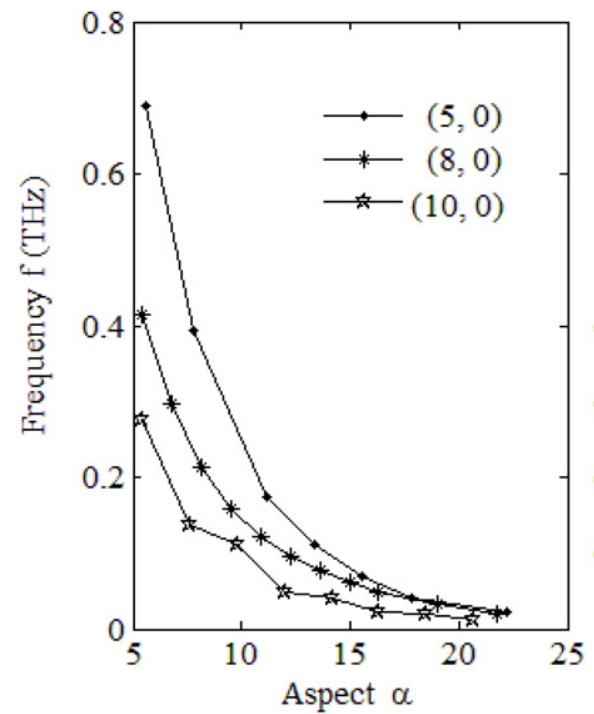

(b)

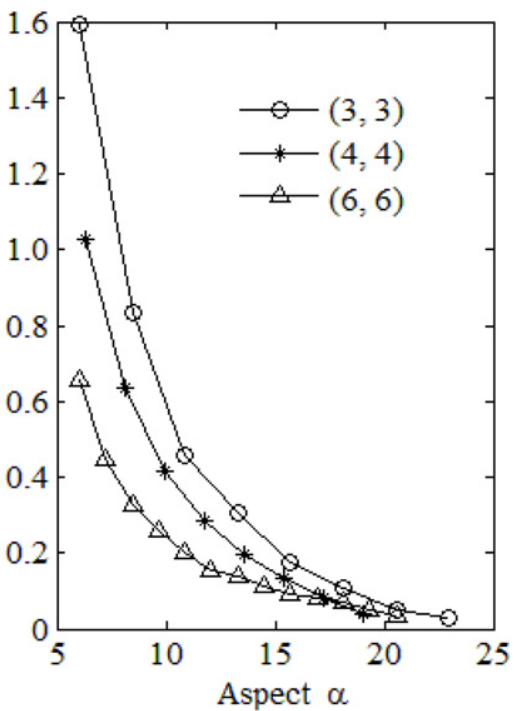

(c)

Figure 6. (a) Transverse vibration of BNNTs, (b) the associated frequency of zigzag BNNTs and (c) the frequency of armchair BNNTs obtained in the MM simulations.

clamped ends have been considered for both zigzag and armchair singlewalled BNNTs with different diameters and aspect ratios. The continuum mechanics models are also employed to account for the physical phenomena observed in the MM simulations. The new findings are summarized as follows.

(1) Thin BNNTs (say, $d<0.65 \mathrm{~nm}$ ) exhibit nearly axisymmetric optimized structures where the crosssection is almost circular and its diameter only varies slightly throughout their length. Nevertheless, the relatively thick BNNTs of sufficiently large aspect ratio (say, $d>0.65 \mathrm{~nm}$ ) display a non-axisymmetric optimized structure characterized by a distorted cross-section with its size varying substantially in axial direction.

(2) In the axial and torsional vibration thin BNNTs behave approximately like uniform columns made of a homogeneous and isotropic material. The equivalent Young's modulus and shear modulus obtained for BNNTs are $1 \mathrm{TPa}$ and $0.4 \mathrm{TPa}$, respectively. The frequency of these thin tubes deceases with increasing length but remains independent of their diameter and chirality. On the other hand, thick BNNTs cannot be treated as uniform columns due to the symmetry-breaking of the optimized configuration.
(3) The radial breathing mode of BNNTs exhibits an approximately linear relation between the associated frequency and the inverse of tube diameter. The coefficient defined by the product of frequency and diameter is found to be sensitive to the constraints imposed on BNNTs. The radial breathing mode of BNNTs thus has the potential to serve as a probe for the tube diameter and end constraints.

(4) In the transverse vibration, the behaviours of BNNTs are qualitatively similar to those of classical beams where the frequency declines with the rising length but increases with the growing size of the tube cross-sections. The constant equivalent bending stiffness $E I$ however cannot be achieved for individual BNNTs mainly due to the sensitivity of $E I$ to the size and shape of the optimized tube cross-section which actually varies in the axial direction for all BNNTs, especially those singlewalled BNNTs with relatively large diameter.

\section{References}

[1] Iijima S 1991 Nature 354 56-8

[2] Ajayan P M and Ebbesen T W 1997 Rep. Prog. Phys. 601025

[3] Blase X, Rubio A, Louie S and Cohen M L 1994 Europhys. Lett. 28 335-40 
[4] Chopra N, Luyken R, Cherrey K, Crespi V, Cohen M, Louie S and Zettl A 1995 Science 269 966-7

[5] Golberg D, Bando Y, Tang C and Zhi C 2007 Adv. Mater. $192413-32$

[6] Won C Y and Aluru N R 2008 J. Phys. Chem. C 112 1812-8

[7] Santosh M, Maiti P K and Sood A K 2009 J. Nanosci. Nanotechnol. 9 5425-30

[8] Yuan J and Liew K M 2008 Nanotechnology 19445703

[9] Xu F, Bando Y, Golberg D, Ma R, Li Y and Tang C 2003 J. Chem. Phys. $1193436-40$

[10] Moon W H and Hwang H J 2004 Physica E 23 26-30

[11] Pokropivny V, Kovrygin S, Gubanov V, Lohmus R, Lohmus A and Vesi U 2008 Physica E 40 2339-42

[12] Li C Y and Chou T W 2006 J. Nanosci. Nanotechnol. 6 54-60

[13] Verma V, Jindal V K and Dharamvir K 2007 Nanotechnology 18435711

[14] Song J, Wu J, Huang Y, Hwang K C and Jiang H 2008 J. Nanosci. Nanotechnol. 8 3774-80

[15] Song J, Wu J, Huang Y and Hwang K C 2008 Nanotechnology 19445705

[16] Jeon G S and Mahan G D 2009 Phys. Rev. B 79085424

[17] Ghassemi H M and Yassar R S 2010 Appl. Mech. Rev. 63020804

[18] Suryavanshi A, Yu M, Wen J, Tang C and Bando Y 2004 Appl. Phys. Lett. 84 2527-9

[19] Chopra N and Zettl A 1998 Solid State Commun. 105 297-300

[20] Zhi C, Bando Y, Tang C, Honda S, Kuwahara H and Golberg D 2006 J. Mater Res. 21 2794-800

[21] Huang Q, Bando Y, Xu X, Nishimura T, Zhi C, Tang C, Xu F, Gao L and Golberg D 2007 Nanotechnology 18485706

[22] Zhi C Y, Bando Y, Tang C C, Huang Q and Golberg D 2008 J. Mater. Chem. 18 3900-8

[23] Oh E S 2010 Mater. Lett. 64 859-62

[24] Hernandez E, Goze C, Bernier P and Rubio A 1998 Phys. Rev. Lett. 80 4502-5
[25] Akdim B, Pachter R, Duan X F and Adams W W 2003 Phys. Rev. B 67245404

[26] Sanchez-Portal D and Hernandez E 2002 Phys. Rev. B 66235415

[27] Wirtz L and Rubio A 2003 IEEE Trans. Nanotechnol. 2 341-8

[28] Zhang Z H, Guo W L and Dai Y T 2009 J. Appl. Phys. 105084312

[29] Jensen K, Kim K and Zettl A 2008 Nat. Nanotechnol. 3 533-7

[30] Li C Y and Chou T W 2004 Appl. Phys. Lett. 84 5246-8

[31] Chowdhury R, Adhikari S and Mitchell J 2009 Physica E 42 104-9

[32] Allen B L, Kichambare P D and Star A 2007 Adv. Mater. 19 1439-51

[33] Balasubramanian K and Burghard M 2006 Anal. Bioanal. Chem. 385 452-68

[34] Frisch M J et al 2009 Gaussian 09 (USA: Gaussian Inc.)

[35] Rappe A K, Casewit C J, Colwell K S, Goddard W A and Skiff W M 1992 J. Am. Chem. Soc. 114 10024-35

[36] Rappé A K and Goddard W A III 1991 J. Phys. Chem. $953358-63$

[37] Mirzaei M and Giahi M 2010 Physica B 405 2542-4

[38] Akdim B, Kim S N, Naik R R, Maruyama B, Pender M J and Pachter P 2009 Nanotechnology 20355705

[39] Timoshenko S, Young D H and Weaver W 1974 Vibration Problems in Engineering (New York: Wiley)

[40] Wang C Y, Ru C Q and Mioduchowski A 2004 J. Appl. Mech. Trans. ASME E 71 622-31

[41] Rao A M et al 1997 Science 275 187-91

[42] Bandow S, Asaka S and Satio Y 1998 Phys. Rev. Lett. 80 3779-82

[43] Kürti J, Kresse G and Kuzmany H 1998 Phys. Rev. B 58 R8869-72

[44] Wirtz L, Rubio A, Concha R A and Loiseau A 2003 Phys. Rev. B 68045425 